\title{
Multiphase transformer-coupled converter: two different strategies for energy conversion
}

\author{
M.C.Gonzalez, P.Alou, O.Garcia, J.A.Oliver, J.A.Cobos \\ Centro de Electrónica Industrial \\ Universidad Politecnica de Madrid \\ Madrid, España \\ Email: carmen.gsanchez@upm.es
}

\begin{abstract}
The operation of a multiphase topology, ideally, without energy storage presents the advantage of achieving very high efficiency over a wide load range as well as a fast dynamic response. However, ideal no-energy storage operation also implies a limitation in the regulation capability of the topology, the output voltage can only take discrete values. These features (high efficiency and discrete regulation capability) of the proposed energy conversion strategy enable the topology as a candidate for 'DC-DC transformer' applications. The advantages, drawbacks and the operating principle of this concept, implemented with a 'closed chain' magnetic structure have been already presented. In this work, the minimum energy storage operation, is applied to two different magnetic structures. These magnetic structures are called 'closed chain' and 'pyramidal'; the main advantage of the 'pyramidal' coupling structure is to improve the size of the converter without increasing the operating frequency. Both magnetic structures are analyzed, compared and experimentally implemented.
\end{abstract}

\section{INTRODUCTION}

A multiphase transformer-coupled topology with (ideally) no energy storage, has proved to achieve a reduction in the output impedance of the converter which allows an improvement in the dynamic response ([1], [2]). In this topology, the coupling among the phases is done by transformers instead of coupled inductors ([3], [4]) in order to minimize the energy storage of the converter; an appropriate control strategy that allows the converter to operate (ideally) without output filter is required. One of the implications of operating a converter with minimum energy storage, is that the operating frequency and the dynamic response of the converter are decoupled. This is the main advantage of this concept, providing very fast dynamic response as well as very high efficiency over a wide load range. However, minimum energy storage operation also implies that the duty cycle can not be variated in a continuous way. This is the main drawback of this concept, the duty cycle can only take discrete steps.

The high efficiency makes this topology a good candidate for a pre-regulator in two-stage power architectures. In this kind of applications, the first stage can be a 'DC/DC transformer' and the final regulation is provided by a point of load converter. Two-stage power architectures can achieve better efficiency and size in comparison with a single stage power architecture if both stages are adequately designed. Examples of two-stage power architectures can be found in [5] and [6].
As said above, in the proposed topology the transfer of the energy and the operating frequency are decoupled; fast dynamics are achieved while keeping very high efficiency ([1]). The decoupling between energy transfer and switching frequency provides a degree of freedom in the design since the operating frequency can be selected to optimize the efficiency without affecting the dynamics of the converter. However, the trade-off between size and efficiency is not changed by the minimum energy storage operation: if the transformers have a larger size, a higher efficiency can be achieved. As usual, the size of the transformers can be reduced by increasing the switching frequency of the converter, always at the cost of degrading efficiency. Examples of different coupling strategies, based on discrete transformers are presented in [7]. One of the magnetic structures presented in [7] is the 'closed chain' magnetic structure; previously, this magnetic structure has been used to implement the minimum energy storage concept which is validated in [1] and [2].

In this paper, a magnetic structure that could enable an improvement in the size of the converter is proposed for the implementation of the minimum energy storage converter. This coupling strategy has been called 'pyramidal' magnetic structure and is based on an arrangement of discrete transformers. With this coupling strategy, the size of the converter can be reduced without increasing the switching frequency; thus it could be possible to reduce the size without degrading the efficiency and the dynamic response. An analysis of this magnetic structure operated under the no energy storage condition is presented in section III and the concept is validated by means of an experimental prototype which is presented in section IV and compared with the 'closed chain' implementation. The efficiencies and dynamic responses obtained with both conversion strategies ('closed chain' and 'pyramidal' magnetic structures) are compared in section IV.

\section{Minimum energy Storage: Concept Review}

In order to achieve minimum energy storage, the coupling between the phases of the converter is done by means of transformers since ideally, transformers do not store energy. Besides, with the aim of operating ideally without output filter, a specific control strategy is required. In figure 1a), a ' $n$ ' phases topology is shown. The control strategy is also 


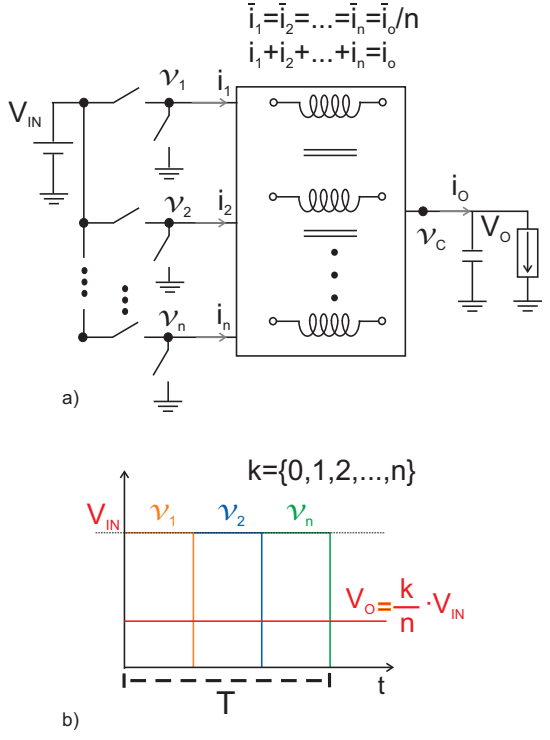

Fig. 1. ' $n$ ' phases converter. a) General magnetic structure. b) proposed control strategy: $v_{1}+v_{2}+\ldots+v_{n}$ must be constant for every instant of time

illustrated in figure 1b). The control strategy must satisfy the following conditions:

- The sum of the voltages at the input of the magnetic structure $\left(\sum v_{i}\right)$ must be constant for every instant of time along a switching cycle. In figure $1, v_{1}+v_{2}+\ldots+v_{n}=$ constant $\forall t$

- The mean value of the voltage in each phase $\left(\bar{v}_{i}\right)$ must be equal among all phases, in order to avoid transformer saturation. Besides, this value $\left(\bar{v}_{i}\right)$ is equal to the output voltage of the converter $\left(V_{O U T}\right)$ since the mean value of the voltage across each transformer of the magnetic structure is zero. In figure $1, \overline{v_{1}}=\overline{v_{2}}=\ldots=\bar{v}_{n}=\bar{V}_{O U T}$

If these conditions are accomplished for every instant of time, the output voltage will be also constant for every instant of time without the need of an output filter. The duty cycles where this control strategy is achieved are called operating nodes. And are given by:

$$
d=k \cdot \frac{1}{n}
$$

where $n$ is the number of the phases and $k$ is an integer, ranging from 0 to $n$. Also, it is necessary that the shifting of the control signals is given by $\frac{360^{\circ}}{n}$.

Regardless the coupling magnetic structure, the output of the converter is given by the following equation:

$$
V_{O U T}=\bar{v}_{i}=d \cdot V_{I N}
$$

and for duty cycles where the sum of the input voltages to the magnetic structure is constant $\left(\sum v_{i}=\right.$ constant $), v_{C}=$ is also constant, and no output inductor $\left(L_{F I L T E R}\right)$ is necessary.

The number of available output voltage values is related to the number of phases; hence, increasing the number of phases in the converter means increasing the available output voltage

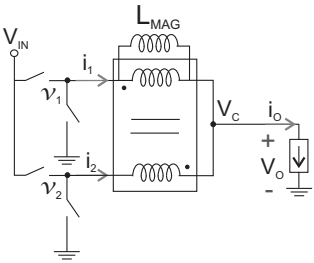

a)
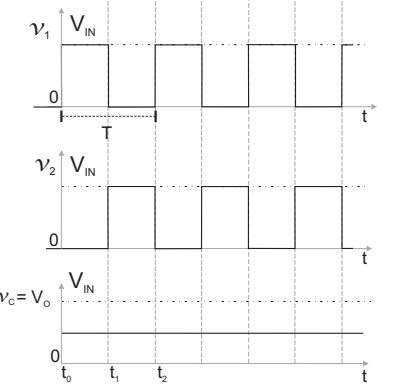

b)

Fig. 2. Example of transformer-coupled topology with two phases

values.

The current sharing is guaranteed by the operation of the topology; the ampere-turns balance makes it possible that the mean value of all phase currents is the same:

$$
\frac{\bar{i}_{\text {OUT }}}{n}=\overline{i_{1}}=\overline{i_{2}}=\ldots=\overline{i_{n}}
$$

and the value of the output current is given by:

$$
i_{\text {OUT }}=i_{1}+i_{2}+\ldots+i_{n}
$$

Total ripple cancellation is assumed.

A two-phase converter is shown in figure 2. For a two-phase converter, there is only one operating node at $50 \%$ duty cycle; so only one output voltage value is available. For every instant of time, output voltage of the transformer $\left(v_{C}\right)$ is given by:

$$
v_{C}=\frac{v_{1}+v_{2}}{2}
$$

and $\overline{v_{1}}=\overline{v_{2}}=\overline{v_{C}}$; with the applied control strategy it is assessed that $v_{1}+v_{2}=V_{I N}$, hence

$$
V_{O U T}=\frac{V_{I N}}{2}
$$

If the magnetizing current is neglected, the ampere-turns balance asses that $i_{1}=i_{2}$ and $i_{O U T}=i_{1}+i_{2}$.

\section{Minimum ENERGy STORAGE: CANDIDATE MAGNETIC STRUCTURES}

Different arrangements of transformers are available in the literature in order to couple the phases of a multiphase converter ([8], [7]). Among these arrangements, 'closed chain' and 'pyramidal' magnetic structures are reported. All these transformer arrangements are used in combination with an output filter. 


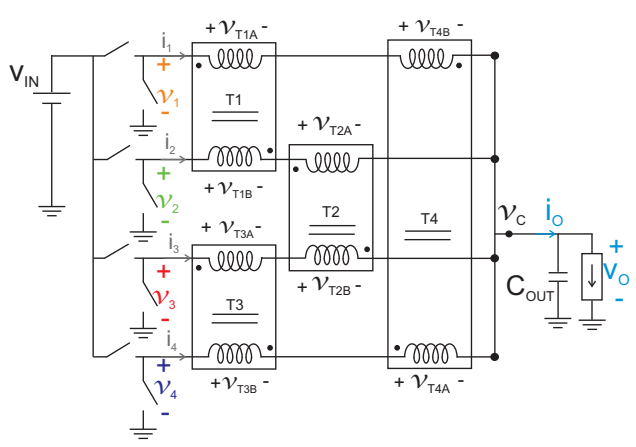

Fig. 3. 'Closed Chain’ magnetic structure (previous)

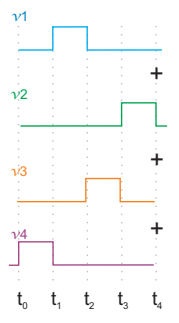

a)

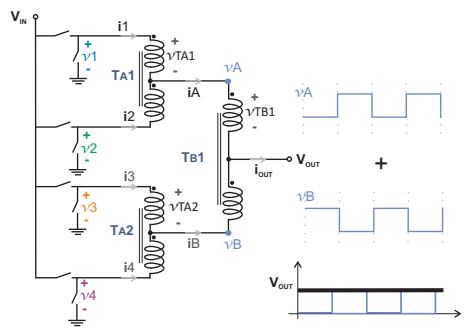

b)
Fig. 4. 'Pyramidal' magnetic structure for four-phase topology

In this paper, both the 'closed chain' magnetic structure (figure 3) and the 'pyramidal magnetic structure' are compared when used for implementing the minimum energy storage converter. Ideally, both magnetic structures are operated without output filter. For both magnetic structures, the particular form of equations 1, 2, 3 and 4 for a four-phase topology are valid. However the way in which the energy is processed inside the magnetic structure does change from one coupling structure to another.

Regarding energy processing, the main characteristic of the 'closed chain' magnetic structure (shown in figure 3 ) is that the current through all the transformers is equal. On the other hand, it can be said that the 'pyramidal' magnetic structure shown in figure $4 \mathrm{~b}$ is composed by different levels. It can be said that, in figure 4 the first level comprises transformers $T_{A 1}$ and $T_{A 2}$ while the second level is composed by transformer $T_{B 1}$. Neglecting magnetizing current, the currents through $T_{A 1}$ and $T_{A 2}$ are equal, but different from the current handled by transformer $T_{B 1}$. The current through $T_{B 1}$ is twice the current through each of the transformers in the first level $\left(T_{A 1}, T_{A 2}\right)$.

The main difference between both coupling strategies is the number and the design of the transformers. For example, a four phase converter implemented with the 'pyramidal' magnetic structure (figure 4b) can be implemented with three transformers; for the implementation of a four phase converter with the 'closed chain' magnetic structure, four transformers are required. The 'pyramidal' magnetic structure can be extended to any power-of-two number of phases; a generalization of the 'pyramidal' magnetic structure, is shown in figure 5.

In order to compare both magnetic structures for the implementation of a transformer-coupled converter with minimum

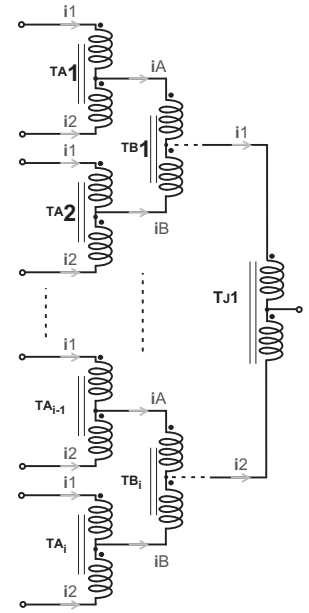

Fig. 5. Generalization of pyramidal magnetic structure

energy storage, an analysis of the steady state operation of each magnetic structure is presented. Both analyses are done in the time domain and under the following assumptions:

- Leakage inductances of the transformer are negligible $\left(L_{L K E Q}=0\right)$.

- The magnetizing inductance $\left(L_{M A G}\right)$ is the same for all the transformers in the converter.

- The converter operates only in the duty cycles given by equation 1 ; for a four-phase converter $(n=4)$, three duty cycles are available: $25 \%, 50 \%, 75 \%$.

The objective of the analysis is to obtain the relationship between the magnetizing inductance of the transformers and the switching frequency of the converter, with this information, a losses analysis can be done for each magnetic structure.

\section{A. Analysis of 'closed chain' magnetic structure}

An analysis of the magnetic structure shown in figure 3, operated with the proposed control strategy is presented. The objective of the analysis is to deduce the magnitude of the phase current ripples, in order to calculate the losses of the magnetic structure. As said before, in this magnetic structure, the average currents seen by all the transformers are equal among them, so it is necessary to deduce only one equation for each phase.

Two particular assumptions are done in order to analyze the closed chain magnetic structure:

- The relation between the voltages of the windings in each transformer is defined by the following equations:

$$
\begin{aligned}
& v_{T 1 A}=-v_{T 1 B} \\
& v_{T 2 A}=-v_{T 2 B} \\
& v_{T 3 A}=-v_{T 3 B} \\
& v_{T 4 A}=-v_{T 4 B}
\end{aligned}
$$

- The nodes at which the converter is operated, coincide with the duty cycles where total ripple cancellation is 
achieved, and it can be assumed that $I_{O U T}$ is constant, hence:

$$
\frac{d i 1}{d t}+\frac{d i 2}{d t}+\frac{d i 3}{d t}+\frac{d i 4}{d t}=0
$$

With these assumptions, the voltage across the transformers can be deduced:

$$
\begin{aligned}
& v_{T 1 A(t)}=\frac{3}{4} v_{1}+\frac{1}{4} v_{3}+\frac{1}{2} v_{4}-\frac{3}{2} v_{C} \\
& v_{T 2 A(t)}=-\frac{1}{4} v_{1}-\frac{3}{4} v_{3}-\frac{1}{2} v_{4}+\frac{3}{2} v_{C} \\
& v_{T 3 A(t)}=-\frac{1}{4} v_{1}+\frac{1}{4} v_{3}+\frac{1}{2} v_{4}+\frac{1}{2} v_{C} \\
& v_{T 4 A(t)}=-\frac{1}{4} v_{1}+\frac{1}{4} v_{3}-\frac{1}{2} v_{4}+\frac{1}{2} v_{C}
\end{aligned}
$$

Where $v_{1}, v_{2}, v_{3}$ and $v_{4}$ stand for the input voltages to the transformers (figure 3 ), and the values of $v_{i}$ are only 0 or $V_{I N}$. Phase ripple due to the magnetizing inductance and the switching frequency of the converter can be expressed as follows:

$$
\begin{gathered}
\Delta I_{M \text { AGphase } 1}=1 / 4 \cdot \frac{\left(v_{T 1 A}-v_{T 3 A}-2 v_{T 4 A}\right) \Delta t}{L} \\
\Delta I_{M A G p h a s e 2}=-1 / 4 \cdot \frac{\left(3 v_{T 1 A}+v_{T 3 A}+v_{T 4 A}\right) \Delta t}{L} \\
\Delta I_{M A G p h a s e 3}=1 / 4 \cdot \frac{\left(v_{T 1 A}+3 v_{T 3 A}+2 v_{T 4 A}\right) \Delta t}{L} \\
\Delta I_{M A G p h a s e 4}=1 / 4 \cdot \frac{\left(v_{T 1 A}-v_{T 3 A}+2 v_{T 4 A}\right) \Delta t}{L}
\end{gathered}
$$

where $v_{T 1 A}, v_{T 2 A}, v_{T 3 A}$ and $v_{T 2 A}$ represent the voltages of the transformers in the magnetic structure (figure 3 ). These ripples are shown in figure 6 for $25 \%$ duty cycle. With these equations, the relation among the magnetizing inductance $\left(L_{M A G}\right)$, the phase ripple and the switching frequency is established, and different designs can be evaluated.

\section{B. Analysis of pyramidal magnetic structure}

Three particular assumptions are done in order to analyze the pyramidal magnetic structure:

- The relation between the voltages of the windings in each transformer is defined by the following equations:

$$
\begin{aligned}
& v_{T A 1}=-v_{T A 1 p} \\
& v_{T A 2}=-v_{T A 2 p} \\
& v_{T B 1}=-v_{T B 1 p}
\end{aligned}
$$

- The currents of the second level of the 'pyramidal' structure $\left(i_{A}, i_{B}\right)$ are obtained by summing the currents in the first level:

$$
\frac{d i 1}{d t}+\frac{d i 2}{d t}=\frac{d i A}{d t}
$$
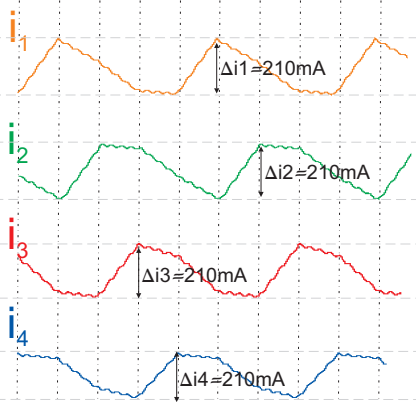

$\begin{array}{lllll}t_{0} & t_{1} & t_{2} & t_{3} & t_{4}\end{array}$

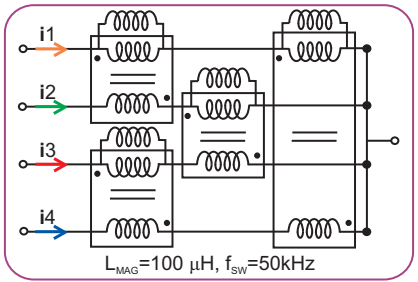

Fig. 6. Magnetizing ripple of phase currents $i_{1}, i_{2}, i_{3}$ and $i_{4}$ in "central chain' magnetic structure

$$
\frac{d i 3}{d t}+\frac{d i 4}{d t}=\frac{d i B}{d t}
$$

- The nodes at which the converter is operated, coincide with the duty cycles where total ripple cancellation is achieved, and it can be assumed that $I_{O U T}$ is constant, hence:

$$
\frac{d i A}{d t}+\frac{d i B}{d t}=0
$$

For this magnetic structure, two set of expressions (one for each level) must be obtained in order to find the ripple in both levels of the pyramid. The first level comprises the transformers $T A 1$ and $T A 2$. The currents of the first level are $i_{1}, i_{2}, i_{3}$ and $i_{4}$ (figure $4 \mathrm{~b}$ ); the second level is formed by transformer $T B 1$ and the currents in this level are $i_{A}$ and $i_{B}$.

The voltages of the transformers $\left(v_{T A 1}, v_{T A 2}\right.$ and $\left.v_{T B 1}\right)$ for the pyramidal magnetic structure can be easily determined taking into account the equation that describes the behavior of the basic cell (fig. 2, eq. 5) and equations 20 through 22. Voltages through the transformers are given by:

$$
\begin{gathered}
v_{T_{A 1}}=1 / 2 \cdot\left(v_{1}-v_{2}\right) \\
v_{T_{A 2}}=1 / 2 \cdot\left(v_{3}-v_{4}\right) \\
v_{T_{B 1}}=1 / 2 \cdot\left(v_{1}+v_{2}\right)-V_{O U T}
\end{gathered}
$$

The ripple of the currents $i_{1}, i_{2}, i_{3}$ and $i_{4}$ (first level) of the pyramidal magnetic structure is a function of the magnetizing inductances in both levels, the voltages of the transformers, the switching frequency of the converter and the duty cycle. The current ripples of the first level are given by: 


$$
\begin{aligned}
& \Delta I_{M A G i 1}=1 / 4 \cdot \frac{\left(2 L_{M A G_{B}} v_{T_{A 1}}+L_{M A G_{A}} v_{T_{B 1}}\right) \Delta t}{L_{M A G_{A}} \cdot L_{M A G_{B}}}(29) \\
& \Delta I_{M A G i 2}=-1 / 4 \cdot \frac{\left(2 L_{M A G_{B}} v_{T_{A 1}}-L_{M A G_{A}} v_{T_{B 1}}\right) \Delta t}{L_{M A G_{A}} \cdot L_{M A G_{B}}} \\
& \Delta I_{M A G i 3}=-1 / 4 \cdot \frac{\left(-2 L_{M A G_{B}} v_{T_{A 2}}+L_{M A G_{A}} v_{T_{B 1}}\right) \Delta t}{L_{M A G_{A}} \cdot L_{M A G_{B}}} \\
& \Delta I_{M A G i 4}=-1 / 4 \cdot \frac{\left(2 L_{M A G_{B}} v_{T_{A 2}}+L_{M A G_{A}} v_{T_{B 1}}\right) \Delta t}{L_{M A G_{A}} \cdot L_{M A G_{B}}}
\end{aligned}
$$

$v_{T A 1}, v_{T A 2}$ and $v_{T B 1}$ are the voltages across the transformers $T A 1, T A 2$ and $T B 1$ respectively. $L_{M A G_{A}}$ and $L_{M A G_{B}}$ represent the magnetizing inductances of transformers in the first and second level of the pyramid, respectively. Since the magnitudes of the ripple across the transformers in the first level ( $T A 1$ and $T A 2)$ are equal, the design of the transformers of this level can be identical. .

Ripple magnitudes in the second level of this magnetic structure, are given by the following equations:

$$
\begin{aligned}
\Delta I_{i A} & =1 / 2 \cdot \frac{v_{T_{B 1}} \Delta t}{L_{M A G_{B}}} \\
\Delta I_{i B} & =-1 / 2 \cdot \frac{v_{T_{B 1}} \Delta t}{L_{M A G_{B}}}
\end{aligned}
$$

$v_{T B 1}$ represents the voltage across the transformer $T B 1$. The mean value of the current across the transformer of level two is $\frac{i_{O U T}}{2}$ while for the transformers in level one the mean value of the current is $\frac{i_{O U T}}{4}$. Due to this factor, the design of the transformers of level one (TA1 and $T A 2)$ can be different from the design of the transformer in level two. It is also important to point out, that in the first level, the value of the ripple is related to the magnetizing inductance of both levels and this has to be taken into account in order to realize a good design. The ripple currents for this magnetic structure are represented in figure 7 for a duty cycle of $25 \%$.

\section{Comparison of both structures}

Losses have been evaluated for both magnetic structures. Using equations (16)-(19) the losses of the 'closed chain' magnetic structure are calculated with the losses model presented in [1]. Also, using this model, the losses are calculated for the 'pyramidal' magnetic structure, using the equations (29)-(34) reported in IV. In order to compare both magnetic structures, the losses are evaluated using the following specifications: Input voltage $12 \mathrm{~V}$, duty cycle $50 \%, L_{M A G}=70 \mu H, f_{S W}=$ $80 \mathrm{kHz}$. The same MOSFETs and drivers are used in order to calculate the losses in both prototypes for a fair comparison, data from IRF7831 MOSFETs and IR2181 drivers has been considered in the losses model.

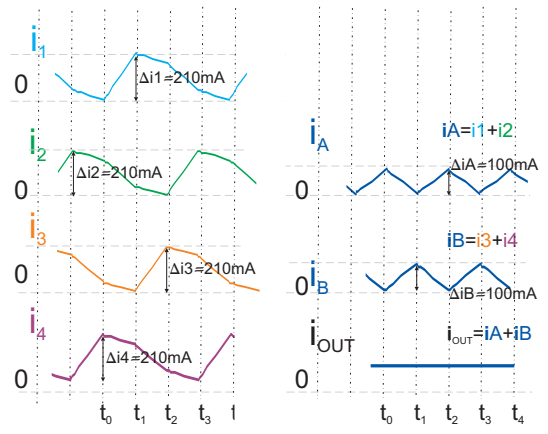

a)

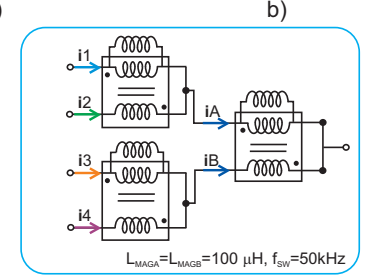

Fig. 7. Currents of both levels of the 'pyramidal' magnetic structure. a)Ripple due to the magnetizing inductances on the first level. b)Ripple due to the magnetizing inductances on the second level.

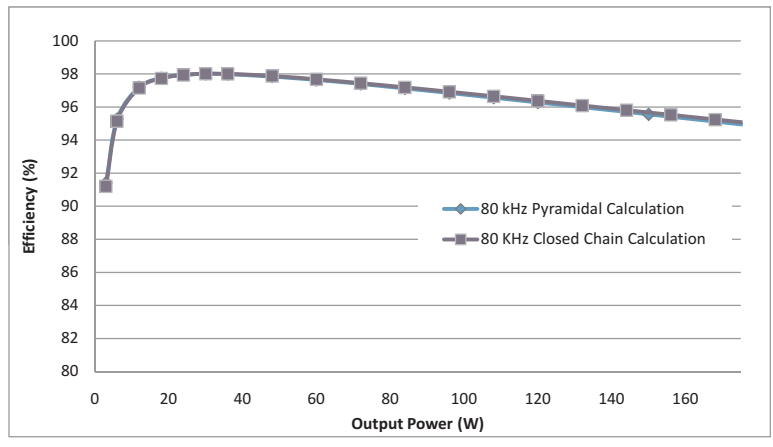

Fig. 8. Power losses calculation for two converters, with the 'closed chain' and 'pyramidal' magnetic structure for $50 \%$ duty cycle

The resulting calculations are shown in the graph in figure 8 . It can be seen that both magnetic structures, result in almost the same losses.

\section{EXPERIMENTAL VALIDATION}

\section{A. Implementation of both magnetic structures}

In order to validate the proposed magnetic structure, both magnetic structure configurations have been tested under the same specifications and with the same transformers. This specifications are: $12 \mathrm{~V}$ input voltage, $6 \mathrm{~V}$ output voltage, $30 \mathrm{~A}$ maximum output current, switching frequency $80 \mathrm{kHz}$, transformers are implemented in a RM6-3F3 core with an $L_{M A G}=70 \mu \mathrm{H}$. The ratio of all the transformers is $1: 1$.

In figure 9, the efficiencies of both magnetic structure for different loads and a switching frequency of $80 \mathrm{kHz}$ are presented when operating at $25 \%$ duty cycles, the achievable power is the same for both magnetic structures. In figure 10, the efficiencies of both magnetic structures, operating with 


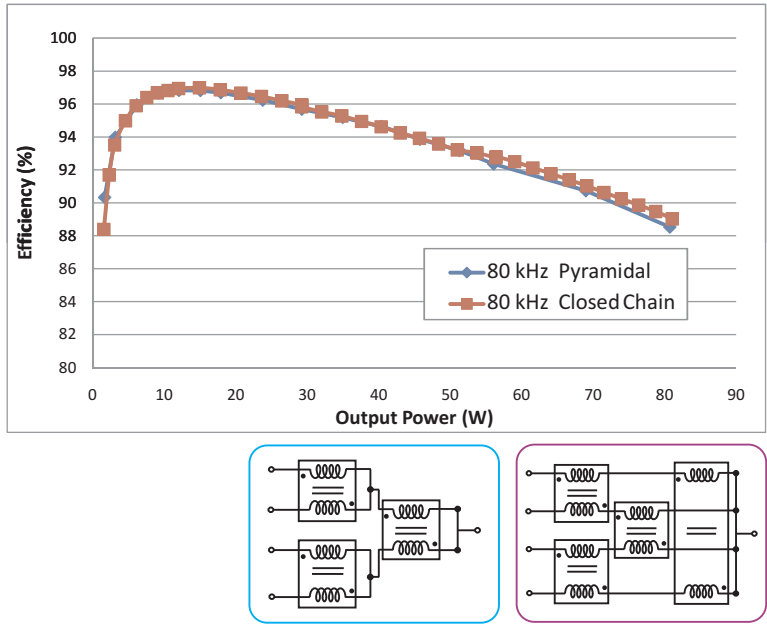

Fig. 9. For $25 \%$ duty cycle and $V_{O U T}=3 \mathrm{~V}$, comparison of the efficiency of 'closed chain' and 'pyramidal' magnetic structures

$50 \%$ duty cycle are shown, it can be seen that the efficiency of both set-ups is very similar. Efficiency from $6 \mathrm{~W}$ to $150 \mathrm{~W}$ is higher than $95 \%$.

The measurements in figure 10 can be compared with the calculations done in previous section. It can be seen that, the losses are predicted with acceptable accuracy, however, in the real prototypes the differences between the efficiencies of both converters are higher.

Dynamic response of the topology with the pyramidal magnetic structure is shown in figure 11. This response is achieved at $3 \mathrm{~V}$ of output voltage and it can be seen that, under a $10 \mathrm{~A}(40 \mathrm{~A} / \mu \mathrm{s})$ load step, the output voltage drop is less than $5 \%$. Output capacitor is formed by 6 ceramic capacitors of $22 \mu \mathrm{F}$ at the output of the converter; input capacitor of the converter for this load step is formed by a $470 \mu F$ OSCON capacitor plus four MLC Capacitors of $22 \mu \mathrm{F}$.

With the same output capacitances and specifications, a $10 \mathrm{~A}$ load step $(40 \mathrm{~A} / \mu \mathrm{s})$ is applied to the 'closed chain' prototype. The response of the converter is shown in figure 12. It can be seen that both responses are very similar.

It is important to point out, that the main difference between both magnetic structures is the number of transformers. While the 'closed chain' structure is composed by four transformers, the pyramidal magnetic structure is composed by three. This represents a reduction of $25 \%$ in the size of the magnetic structure.

\section{B. Design example for efficiency optimization}

The two-phase converter, shown in figure $2 \mathrm{a}$, can be considered to be the basic cell of both magnetic structures (pyramidal and closed chain). This basic cell has been used for the design and implementation of a two-phase converter with very high efficiency in a very wide load range. The specifications of this design are: input voltage $12 \mathrm{~V}$, output voltage $6 \mathrm{~V}$, operating frequency $60 \mathrm{kHz}$, output current $60 \mathrm{~A}$. Since it is a two-phase converter, the duty cycle is fixed to $50 \%$. The

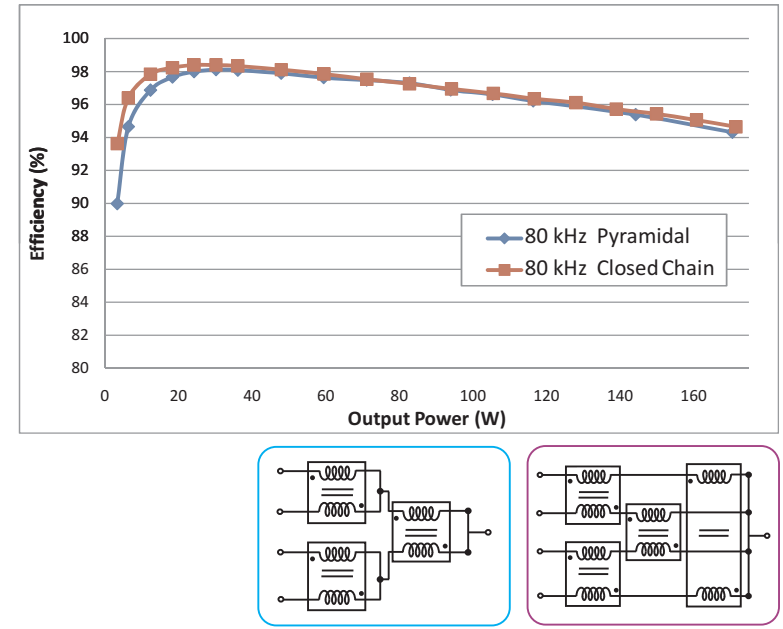

Fig. 10. For $50 \%$ duty cycle and $V_{O U T}=6 \mathrm{~V}$, comparison of the efficiency of 'closed chain' and 'pyramidal' magnetic structures.

selected MOSFETs are PSMN1R2-25YL, for both the control and synchronous rectifier switch, and the driver is IR2181. The magnetic structure is build with a 25.3/14.8/10 - N27 EPCOS toroid. The aforementioned losses model (reported in [1]) is used in order to design a converter where the equivalent series resistance is minimized. The selected MOSFETs and the design of the magnetic structure have been adressed with this model in order to reduce the conduction resistance which is the main cause of the losses at high loads. A picture of the converter is shown in figure 13. Efficiency measurements of the converter are shown in figure 14. It can be seen that the efficiency is almost $98 \%$ from $90 \mathrm{~W}$, to $360 \mathrm{~W}$ ( $15 \mathrm{~A}$ to $60 \mathrm{~A}$ ).

\section{CONCLUSION}

In this paper, the minimum energy storage conversion strategy based on a pyramidal magnetic structure is presented, analyzed and compared with a previous magnetic structure. The main feature of this concept is that, with the use of transformers as a coupling element and an adequate control strategy, the converter is operated with minimum energy storage. Main advantage of minimum energy storage operation is that the dynamic response of the converter and the switching frequency are decoupled. Hence, with this topology it is possible to obtain very high efficiency along with a fast dynamic response.

The main advantage of the 'pyramidal' magnetic structure compared with the 'closed chain', is that a reduction in the size of the converter can be achieved without increasing the operating frequency. Also, in the 'pyramidal' magnetic structure the placement of the transformers is symmetric which improves a reduction in the associated parasitics (series resistance associated to PCB tracks, inductance associated to connexions, etc). In the experimental prototypes presented in Section IV, a reduction of around $25 \%$ in the size of the magnetic structure is achieved when using the 'pyramidal magnetic' structure. The efficiency of the prototype is very 


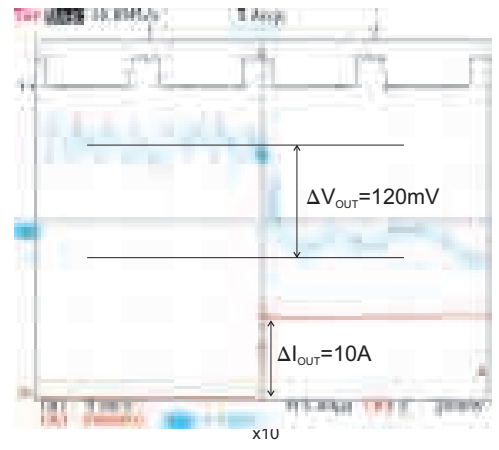

Fig. 11. Dynamic response obtained with pyramidal magnetic structure

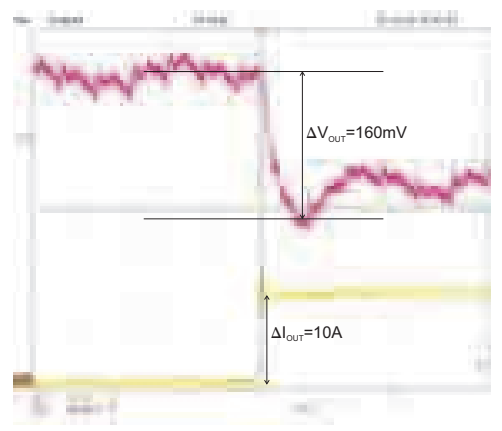

Fig. 12. Dynamic response obtained with closed chain magnetic structure

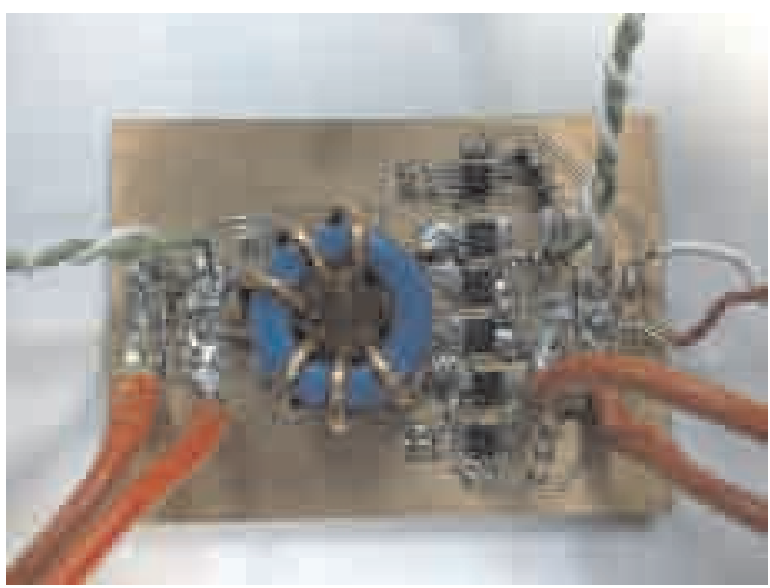

Fig. 13. Picture of a two-phase prototype with 25.3/14.8/10 - N27 EPCOS toroid and designed for high efficiency in a wide load range.

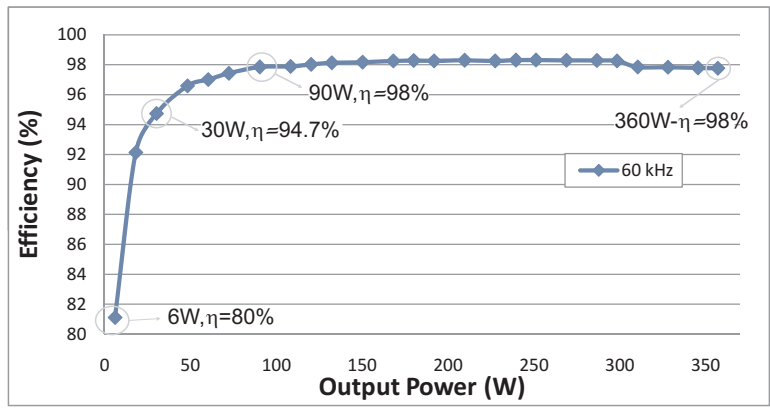

Fig. 14. Efficiency measurements for $50 \%$ duty cycle and $V_{O U T}=6 \mathrm{~V}$ for the two-phase prototype high in a wide load range: from $6 \mathrm{~W}$ to $150 \mathrm{~W}$ efficiency is higher than $95 \%$ (for $50 \%$ duty cycle and $6 \mathrm{~V}$ of output voltage). Compared with the previous magnetic structure, efficiency and dynamic response are very similar. In order to obtain the same dynamic response with an equivalent buck converter, around $100 \mathrm{kHz}$ bandwidth would be needed. A converter with this bandwidth would need around $500 \mathrm{kHz}$ of switching frequency. In contrast, the proposed converter operates in open loop and with $40 \mathrm{kHz}$ of switching frequency, this contributes to reduce the switching losses.

\section{REFERENCES}

[1] M. Gonzalez, P. Alou, O. Garcia, J. Oliver, J. Cobos, and H. Visairo, "Dc-dc transformer multiphase converter with transformer coupling for two-stage architecture," in Applied Power Electronics Conference and Exposition (APEC), 2010 Twenty-Fifth Annual IEEE, 21-25 2010, pp. $781-786$.

[2] M. Gonzalez, L. Laguna, P. Alou, O. Garcia, J. Cobos, and H. Visairo, "New control strategy for energy conversion based on coupled magnetic structures," in Power Electronics Specialists Conference, 2008. PESC 2008. IEEE, 15-19 2008, pp. $704-710$

[3] P.-L. Wong, P. Xu, P. Yang, and F. Lee, "Performance improvements of interleaving vrms with coupling inductors," Power Electronics, IEEE Transactions on, vol. 16, no. 4, pp. 499-507, Jul 2001.

[4] J. Li, C. Sullivan, and A. Schultz, "Coupled-inductor design optimization for fast-response low-voltage dc-dc converters," in Applied Power Electronics Conference and Exposition, 2002. APEC 2002. Seventeenth Annual IEEE, vol. 2, 2002, pp. 817-823 vol.2.

[5] J. Sun, M. Xu, Y. Ying, and F. Lee, "High power density, high efficiency system two-stage power architecture for laptop computers," in Power Electronics Specialists Conference, 2006. PESC '06. 37th IEEE, 18-22 2006, pp. $1-7$.

[6] Y. Ren, M. Xu, K. Yao, Y. Meng, and F. Lee, "Two-stage approach for 12-v vr," Power Electronics, IEEE Transactions on, vol. 19, no. 6, pp. 1498 - 1506, nov. 2004.

[7] P. Zumel, O. Garcia, J. Cobos, and J. Uceda, "Tight magnetic coupling in multiphase interleaved converters based on simple transformers," in Applied Power Electronics Conference and Exposition, 2005. APEC 2005. Twentieth Annual IEEE, vol. 1, 6-10 2005, pp. 385 - 391 Vol. 1.

[8] R. M. P. Anatoli V. Ledenev, Gennady G. Gurov, "Multiple power converter system using combining transformers," Patent 6545 450, 2000. 